\title{
Effect of Constitutive Equations on Prediction Accuracy for Springback in Cold Stamping of TRIP1180
}

\author{
Ki-Young Seo ${ }^{1}$, Jae-Hong Kim ${ }^{1}$, Hyun-Seok Lee ${ }^{2}$, Ji Hoon Kim ${ }^{3}$ and Byung-Min Kim ${ }^{3, *}$ \\ 1 Division of precision and manufacturing systems, Pusan National University, Busandaehak-ro 63beon-gil, \\ Geumjeong-gu, Busan 609-735, Korea; seokiyoung11@pusan.ac.kr (K.Y.S.); kjh86@pusan.ac.kr (J.H.K.) \\ 2 Press Die Research Team, Nara Mold \& Die Co. LTD., Gongdan-ro 675, Seongsan-gu, Changwon-City, \\ Gyeongnam 642-120, Korea; hslee@naramnd.com \\ 3 School of Mechanical Engineering, Pusan National University, Busandaehak-ro 63beon-gil, Geumjeong-gu, \\ Busan 609-735, Korea; kimjh@pusan.ac.kr \\ * Correspondence: bmkim@pusan.ac.kr; Tel.: +82-51-510-2319, FAX.: +82-51-581-3075
}

\begin{abstract}
The objective of this study is to evaluate the effect of constitutive equations on the prediction accuracy for springback in cold stamping with various deformation modes. In this study, two types of yield functions-Hill'48 and Yld2000-2d-were considered to describe yield behavior. Isotropic and kinematic hardening models based on the Yoshida-Uemori model were also adopted to describe hardening behavior. Various material tests (such as uniaxial tension, tension- compression, loading-unloading, and hydraulic bulging tests) were carried out to determine the material parameters of the models. The obtained parameters were implemented in the finite element (FE) simulation to predict springback, and the results were compared with experimental data. U-bending and T-shape drawing were employed to evaluate the springback prediction accuracy. Obviously, the springback prediction accuracy was greatly influenced by constitutive equations. Therefore, it is important to choose appropriate constitutive equations for accurate description of material behaviors in FE simulation.
\end{abstract}

Keywords: advanced high strength steel; yield function; hardening model; springback; deformation mode

\section{Introduction}

In recent years, lightweight vehicles have gained much attention in light of increasingly stringent fuel efficiency and gas emission regulations [1]. Therefore, reducing weight is a key target, and many researchers have devoted significant effort to materials selection for manufacturing automotive parts. Advanced high strength steel (AHSS) is widely used in the automotive industry for its light weight, crashworthiness, and productivity. However, it is difficult to achieve dimensional accuracy because of the excessive springback caused by the higher elastic recovery and high yield strength of AHSS [2]. Achieving a target product shape with AHSS is challenging for part manufacturers, requires considerable amount of time, and involves additional costs to modify tools for springback modification.

Finite element (FE) simulation is applied to describe the material behavior and springback of AHSS, because it provides a cost-effective and reliable method for springback prediction. In FE simulations, constitutive equations strongly affect the accuracy of prediction results. Therefore, various constitutive equations have been suggested by many researchers. The hardening behavior of AHSS has been described by various equations, such as the nonlinear kinematic hardening model proposed by Chaboche [3], the kinematic hardening model based on cyclic plasticity suggested by Yoshida, and the distortional hardening model recommended by Barlat. In addition, Hill' 48 and Yld2000-2d are widely used to model the anisotropic behavior of AHSS. 
The influence of constitutive models on prediction results has also been of interest in many related studies. Lee et al. [4] performed a springback evaluation of automotive sheets based on an isotropic-kinematic hardening model and anisotropic yield functions. In addition, Zang et al. [5] and Larsson et al. [6] employed a combined isotropic-kinematic hardening model to evaluate springback effects in steel sheets. Eggertsen et al. [7] predicted springback using various hardening models and yield functions. Previous studies showed that it is important to describe anisotropic behavior, the Bauschinger effect, transient behavior, and the permanent softening effect. Successful springback prediction by FE simulation is principally dependent on the selection of a yield criterion, a hardening model, and material parameters [8]. However, the above studies dealt with simply configured products, such as those used in U-bending tests [9], and did not focus on AHSS products with various deformation modes. Therefore, it is necessary to evaluate the effect of the constitutive equation on springback prediction accuracy in the cold stamping of AHSS with deformation modes.

The objective of this study is to evaluate the effect of constitutive equations on springback prediction accuracy in the cold stamping of TRIP1180. In this study, two types of yield functions were considered to describe yield behavior: Hill'48 and the Yld2000-2d. Isotropic and kinematic hardening models based on the Yoshida-Uemori model were also adopted to express hardening behavior. Various material tests, such as uniaxial tension, tension-compression, loading-unloading and hydraulic bulging tests were carried out to determine the material parameters of the models. The obtained parameters were implemented into an FE simulation to predict springback, and the results were compared with experimental data. U-bending and T-shape drawing were employed to evaluate the accuracy of the springback prediction.

\section{Constitutive equations for the TRIP1180 sheet steel}

In this study, a TRIP1180 sheet was used as the testing material. Hill'48 [10] and Yld2000-2d [11] were considered to describe yield behavior. Additionally, isotropic and kinematic hardening models based on the Yoshida-Uemori model were adopted to express hardening behavior. The material constants of TRIP1180 for the constitutive equations were obtained from uniaxial tension, tensioncompression, loading-unloading, and hydraulic bulging tests.

\subsection{Yield function}

The yield function defines the transition of a material between elastic and plastic behavior under complex stress states. In this study, Hill'48 and Yld2000-2d yield functions were applied to consider the anisotropic yield behavior of TRIP1180. In order to determine the material parameters of the yield functions, uniaxial tension tests were performed for different rolling directions $\left(0^{\circ}, 45^{\circ}\right.$, $90^{\circ}$ ), and a hydraulic bulge test was performed. The results of these tests are shown in Fig. 1

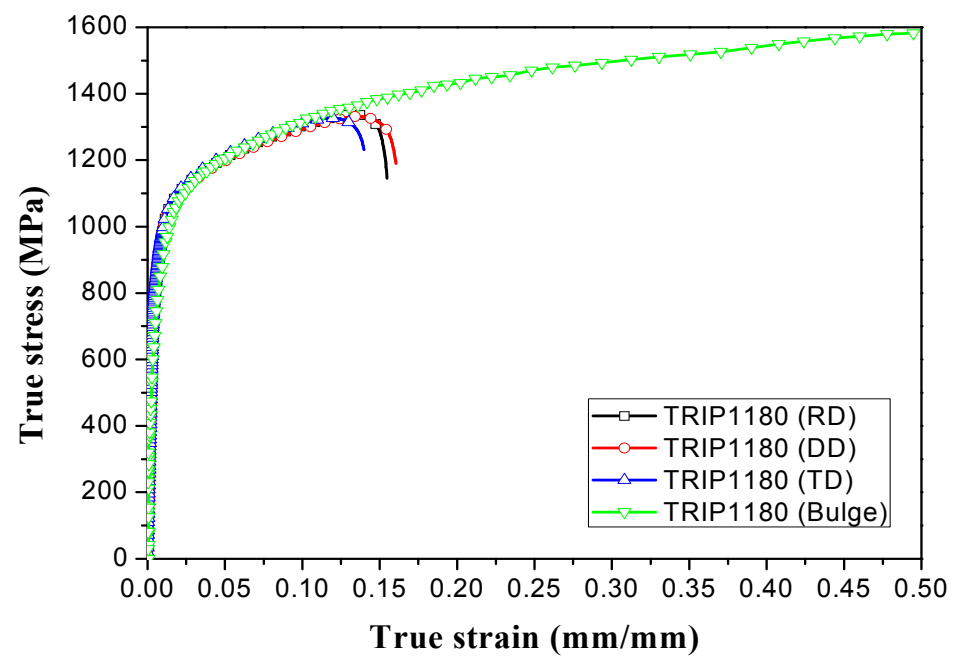

Figure 1. Results of uniaxial tension and bulge tests. 


\subsubsection{Hill' 48 yield function}

The well-known anisotropic yield criterion proposed by Hill is one of the most widely used yield functions. The Hill' 48 yield function is also easy to express, so it has been used widely to investigate the effect of anisotropy on springback, especially for steel sheets. This function is defined as follows:

$$
2 f(\sigma)=\mathrm{F}\left(\sigma_{y y}-\sigma_{z z}\right)^{2}+G\left(\sigma_{z z}-\sigma_{x x}\right)^{2}+H\left(\sigma_{x x}-\sigma_{y y}\right)^{2}+2\left(\mathrm{~L} \sigma_{y z}^{2}+\mathrm{M} \sigma_{z x}^{2}+\mathrm{N} \sigma_{x y}^{2}\right)=1
$$

Under plane stress conditions $\left(\sigma_{z z}=\sigma_{y z}=\sigma_{z x}=0, \mathrm{~L}=\mathrm{M}=0\right)$, the Hill'48 model can be mathematically represented as follows.

$$
2 f(\sigma)=(\mathrm{G}+\mathrm{H}) \sigma_{x x}^{2}+(\mathrm{F}+\mathrm{H}) \sigma_{y y}^{2}-2 H \sigma_{x x} \sigma_{y y}+2 \mathrm{~N} \sigma_{x y}^{2}=1
$$

where $\sigma_{x x}, \sigma_{y y}$, and $\sigma_{z z}$ are normal stresses in the rolling, transverse, and thickness directions, respectively; $\sigma_{x y}, \sigma_{y z}$, and $\sigma_{z x}$ are shear stresses in the $x y, y z$, and zx planes, respectively; and F, G, H, and $\mathrm{N}$ are the anisotropic coefficient parameters. The material parameters of the Hill' 48 yield function are principally obtained from Lankford values at angles of $0^{\circ}, 45^{\circ}$, and $90^{\circ}$ to the rolling direction. The mechanical properties and determined material constants are summarized in Tables 1 and 2.

Table 1. Determined material properties of TRIP1180 steel.

\begin{tabular}{lccccc} 
Test direction & E0 (GPa) & YS (MPa) & UTS (MPa) & Elongation (\%) & R-value \\
\hline ng direction $\left(0^{\circ}\right)$ & 200.5 & 861.9 & 1180 & 17.2 & 0.795 \\
onal direction $\left(45^{\circ}\right)$ & 200.7 & 866.6 & 1175 & 16.0 & 0.958 \\
sverse direction $\left(90^{\circ}\right)$ & 206.3 & 866.2 & 1182 & 14.9 & 0.967 \\
\hline
\end{tabular}

Table 2. Material constants of TRIP1180 for the Hill'48 yield function.

\begin{tabular}{ccccc}
\hline Material & F & G & H & N \\
\hline TRIP1180 & 0.4580 & 0.5571 & 0.4429 & 1.480 \\
\hline
\end{tabular}

\subsubsection{Yld2000-2d yield function}

Yld2000-2d, which was proposed by Barlat et al., can describe yield behavior in various deformation modes. This yield function has eight anisotropic coefficients, which are related to the experimental yield stresses $\left(\sigma_{0}, \sigma_{45}, \sigma_{90}, \sigma_{b}\right)$ and anisotropic parameters $\left(\mathrm{r}_{0}, \mathrm{r}_{45}, \mathrm{r}_{90}, \mathrm{r}_{\mathrm{b}}\right)$. This function can be expressed as shown in Eq. (3):

$$
\phi=\left|X_{1}^{\prime}-X_{2}^{\prime}\right|^{a}+\left|2 X_{2}^{\prime \prime}+X_{1}^{\prime \prime}\right|^{a}+\left|2 X_{1}^{\prime \prime}+X_{2}^{\prime \prime}\right|^{a}=2 \bar{\sigma}
$$

Where $\bar{\sigma}$ is the effective stress and a is a constant related to the crystal structure of the material, which is set to 6 in this study. $X^{\prime}$ and $X^{\prime \prime}$ are the principal values for the symmetric stress, which can be linearly transformed via a relationship equation, as shown in Eq. (4):

$$
X^{\prime}=L^{\prime} \cdot \sigma, X^{\prime \prime}=L^{\prime \prime} \cdot \sigma
$$


Material constants included in the $\mathbf{L}^{\prime}$ and $\mathbf{L}^{\prime \prime}$ tensors can be determined according to the rolling direction using the yield stress and anisotropic coefficient. The determined material constants are summarized in Table 3.

Table 3. Material constants of TRIP1180 for the Yld2000-2d yield function.

\begin{tabular}{cccccccc}
\hline $\boldsymbol{\alpha}_{1}$ & $\boldsymbol{\alpha}_{2}$ & $\boldsymbol{\alpha}_{3}$ & $\boldsymbol{\alpha}_{4}$ & $\boldsymbol{\alpha}_{5}$ & $\boldsymbol{\alpha}_{6}$ & $\boldsymbol{\alpha}_{7}$ & $\boldsymbol{\alpha}_{8}$ \\
\hline 0.9471 & 1.0199 & 0.9867 & 0.9925 & 1.0141 & 0.9815 & 0.9910 & 1.0007 \\
\hline
\end{tabular}

\subsection{Hardening Model}

In plasticity, the hardening rule is used to describe the behavior of a material during plastic deformation. In this study, isotropic and Yoshida-Uemori kinematic hardening models were applied to consider the hardening behavior of TRIP1180. In order to determine the material constants of the hardening models, loading-unloading tests and tension-compression tests were performed. The results are shown in Figs. 2 and 3.

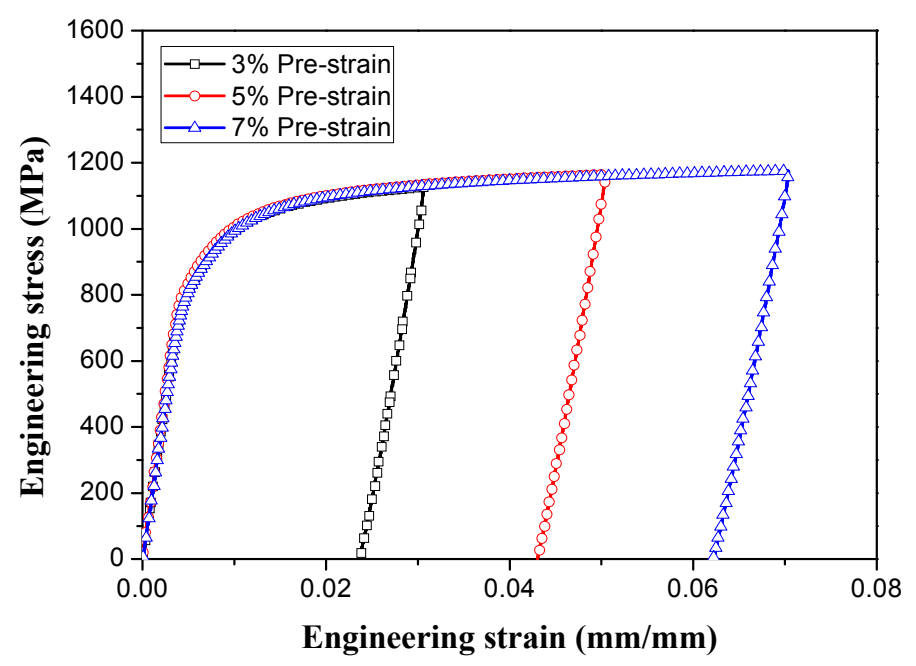

Figure 2. Results of loading-unloading test.

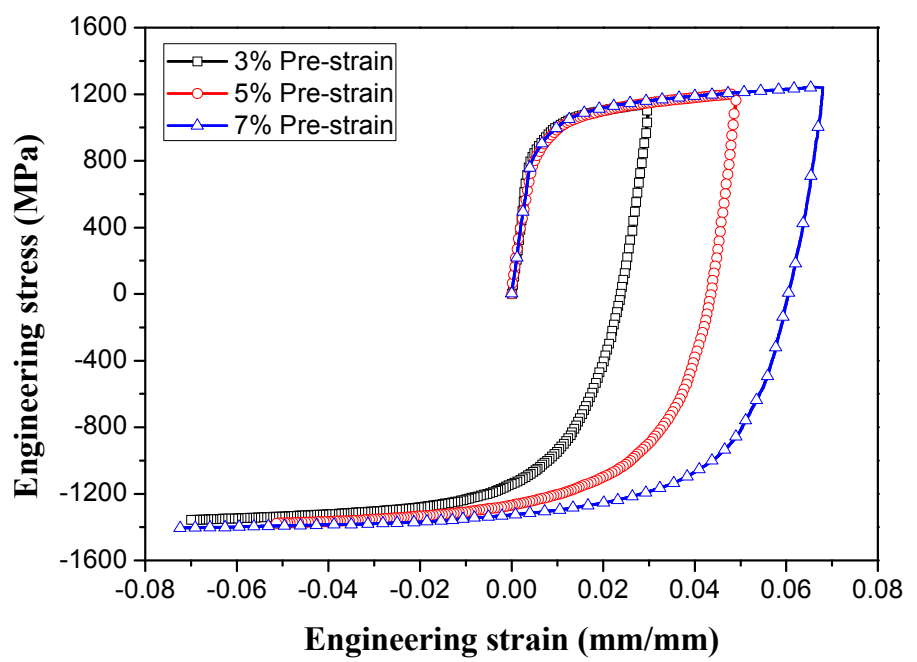

Figure 3. Results of the tension-compression test. 


\subsubsection{Isotropic hardening model}

When expansion is uniform in all directions in a stress space, the hardening behavior is referred to as isotropic. The Swift isotropic hardening model [12] used in this study can be used to successfully describe isotropic behavior. The Swift isotropic hardening model is defined as follows:

$$
\bar{\sigma}=K\left(\bar{\varepsilon}_{0}+\bar{\varepsilon}_{p}\right)^{n}
$$

where $\bar{\sigma}$ and $\overline{\varepsilon_{p}}$ are the effective stress and strain, respectively. $\mathrm{K}, \mathrm{n}$, and $\varepsilon_{0}$ are material constants related to hardening behavior. The material parameters of the Swift hardening model are principally obtained by uniaxial tensile tests, and the determined material constants are summarized in Table 4 .

Table 4. Coefficients of the Swift isotropic hardening model for TRIP1180.

\begin{tabular}{cccc}
\hline Material & K & $\mathbf{n}$ & $\mathcal{E}_{0}$ \\
\hline TRIP1180 & 1.6728 & 0.1044 & $4.8605 \mathrm{E}-14$ \\
\hline
\end{tabular}

\subsubsection{Yoshida-Uemori Hardening Model}

Since material behavior is quite complex during cyclic loading, a hardening rule should accurately predict deformation behavior during cyclic loading. The Yoshida-Uemori model [13] is one of the most sophisticated models, and is capable of reproducing transient Bauschinger effects, permanent softening, and work hardening stagnation in large elasto-plastic deformation. Moreover, the degradation of the elastic modulus during plastic loading is also considered.

The Yoshida-Uemori model takes into account both the translation and expansion of the bounding surface, while the active yield surface evolves in a kinematic manner. The relative displacement of the two yield surface in a bounding surface can be defined as follows:

$$
\alpha_{*}=\alpha-\beta
$$

where $\alpha$ refers to the current center for the yield surface, $\beta$ refers to the center of the bounding surface, and $\alpha_{*}$ refers to the relative position of the two surfaces. $\alpha_{*}$ is also defined in Eq. (7), which determines the relative movement of the yield and bounding surfaces.

$$
\begin{aligned}
& \alpha_{*}=C\left[\left(\frac{a}{Y}\right)(\sigma-\alpha)-\sqrt{\frac{a}{\bar{\alpha}_{*}}} \alpha_{*}\right] \dot{\bar{\varepsilon}} \\
& a=B+R-Y
\end{aligned}
$$

where $B$ refers to the initial size of the bounding surface, $R$ refers to the isotropic hardening component, $Y$ refers to the initial yield strength, and $C$ refers to a material parameter for the kinematic yield surface hardening rule. Isotropic and kinematic hardening behaviors in the bounding surface can be defined as follows:

$$
\begin{gathered}
d R=m\left(R_{s a t}-R\right) \dot{\bar{\varepsilon}} \\
d \beta=m\left(\frac{2}{3} b D^{p}-\beta\right) \dot{\bar{\varepsilon}}
\end{gathered}
$$

where $R_{\text {sat }}$ is the saturated value of the isotropic hardening stress $R$ for infinitely large plastic strain, and $\mathrm{m}$ is a material parameter that controls the rate of isotropic hardening. $D^{p}$ refers to an increment of the plastic deformation rate and $b$ refers to a material constant. The Yoshida-Uemori model constants in the determined TRIP1180 sheets are presented in Table 5.

Generally, the elastic modulus of steel sheets is reduced as the effective strain increases [14-15]. This phenomenon can be formulated as shown in Eq. (10). 


$$
E=E_{0}-\left(E_{0}-E_{a}\right)\left[1-\exp \left(-\xi \varepsilon_{p}\right)\right]
$$

where $E_{0}$ and $E_{a}$ represent the elastic modulus values at the initial state and pre-strain state of the material, respectively. $\xi$ is a material parameter related to softening behavior. The optimized constants are given in Table 6.

Table 5. Material constants of TRIP1180 for the Yld2000-2d yield function.

\begin{tabular}{cccccccc}
\hline $\boldsymbol{B}$ & $\boldsymbol{B}$ & $\boldsymbol{R}_{\text {sat }}$ & $\boldsymbol{b}$ & $\boldsymbol{m}$ & $\boldsymbol{C}_{1}$ & $\boldsymbol{C}_{2}$ & $\boldsymbol{\varepsilon}_{p, \text { ref }}$ \\
\hline 800 & 284.9 & 294.2 & 88 & 9.62 & 366.8 & 366.8 & 0.005 \\
\hline
\end{tabular}

Table 6. Material constants of TRIP1180 for softening behavior.

\begin{tabular}{cccc}
\hline Material & $\boldsymbol{E}_{0}$ & $\boldsymbol{E}_{\boldsymbol{a}}$ & $\xi$ \\
\hline TRIP1180 & 202.1 & 168.7 & 72.9 \\
\hline
\end{tabular}

\section{Test conditions}

In this study, FE simulations and experiments were performed for various forming processes, such as U-bending and T-shape drawing. A commercial program (PamStamp 2G) was used to perform the FE simulations. Forming and springback analyses were conducted using various constitutive equations, the material constants of which were determined in Chapter 2.

\subsection{U-bending test}

The U-bending test has been approved as a significant verification model for springback prediction, as reported in several works. Fig. 4 shows the tools used in U-bending, which consist of a punch, blank holder, and die. The blank is $300.0 \mathrm{~mm}$ long $\times 30.0 \mathrm{~mm}$ wide $\times 1.1 \mathrm{~mm}$ thick. The gap between the die and the punch was designed to be $1.2 \mathrm{~mm}$. Testing was carried out with a 200 ton servo press machine. The total punch stroke was $60.0 \mathrm{~mm}$, with a punch speed of $1 \mathrm{~mm} / \mathrm{s}$ and a blank holding force of $20 \mathrm{kN}$. Tests were also performed for various rolling directions $\left(0^{\circ}, 45^{\circ}, 90^{\circ}\right)$. In addition, experiments for each condition were carried out five times to ensure the reliability of the experiment. After stamping, the final dimensions of the formed specimens were measured along the middle cross section using a laser coordinate measuring machine, to allow for comparison with the results of the FE simulation.

To evaluate springback behavior using a U-bending test, FE simulations were carried out for forming and springback analysis. The analytical model was applied in the same way as in the experiment, and a half model of the tools and blank was simulated, considering the geometric symmetry of the test, as shown in Fig. 5. The specimen for the FEA was a Belytschko-Lin-Tsay (BLT) shell element of uniform size $(1.0 \times 1.0 \mathrm{~mm})$ with five integration points in the thickness direction. The die was assumed to be a rigid body. The FE simulation conditions were identical to the experimental conditions. The Coulomb friction coefficient between the die and specimen was set as 0.12 , a value that assumes unlubricated conditions. In the FE simulation, mass-scaling and mesh-refinement techniques were applied to ensure the efficiency of the analysis. The shapes of the specimens calculated in FE simulations were compared with those from the experimental results [16]. 

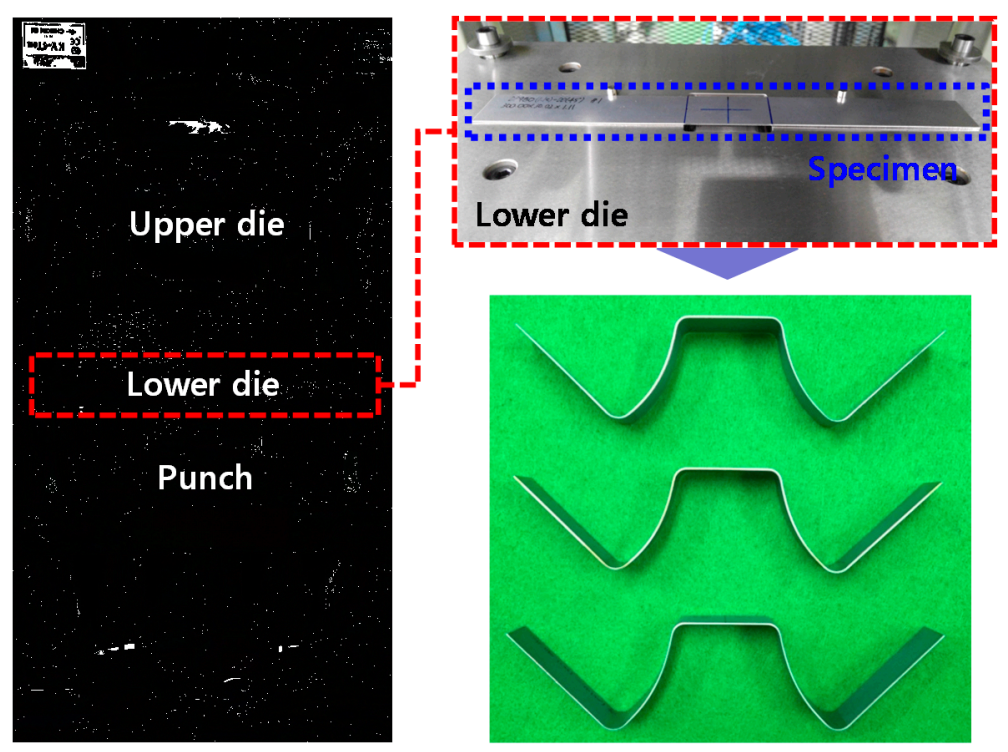

Figure 4. Tools for U-bending test.

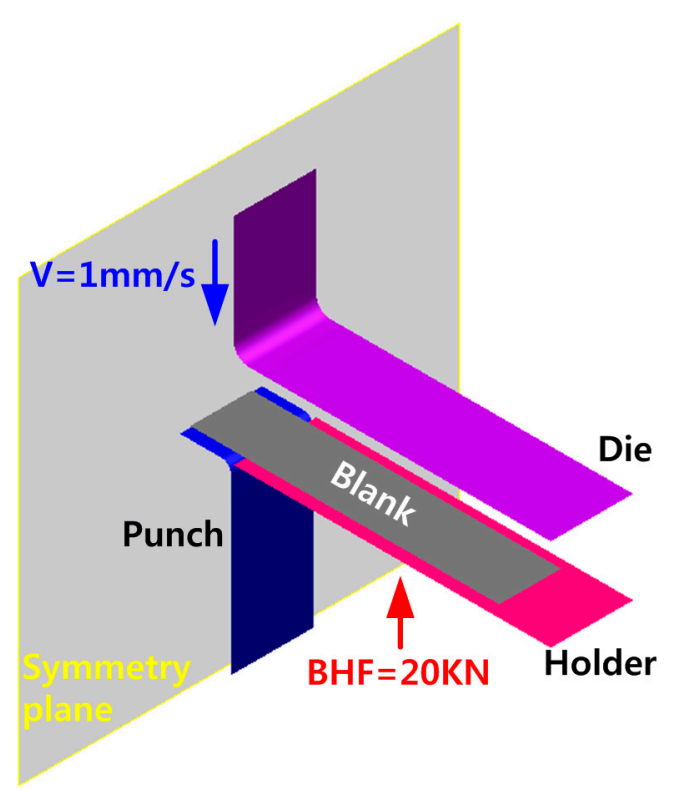

Figure 5. FE model for U-drawing/bending.

\subsection{T-shape drawing test}

In this study, a T-shape drawing test was performed to evaluate the effect of the constitutive equation on the prediction accuracy of springback for complex deformation modes. Fig. 6 shows the experimental set-up for the T-shape drawing test, which consists of a punch, blank holder, and die. The gap between the die and punch was designed to be $1.1 \mathrm{~mm}$, and the test was carried out with a 200-ton servo press machine. The total punch stroke was $22.0 \mathrm{~mm}$, with a punch speed of $20 \mathrm{~mm} / \mathrm{s}$ and blank holding force of $90 \mathrm{kN}$. In addition, experiments were carried out five times to ensure reliability. After stamping, the final dimensions of the formed specimens were measured using a 3D optical scanning system, to allow for comparison with the results of the FE simulation.

To investigate springback behavior during a T-shape drawing test, FE simulations were carried out for forming and springback analysis. The analytical model was applied in the same way as in the experiments; the FE model is shown in Fig. 7. A significant number of conditions for the FE simulation were the same as in the FE simulation of the U-bending test and experimental conditions of the T-shape drawing test. The shapes of the specimens determined through FE simulations were compared with those obtained from experimental results. In this study, Geomagic Design X, which 
is a commercial reverse-design program, was employed to quantitatively compare the configurations. For comparing configurations, the experimental results were input as a reference configuration in order to measure the error in dimensions between the experimental and analytical results.
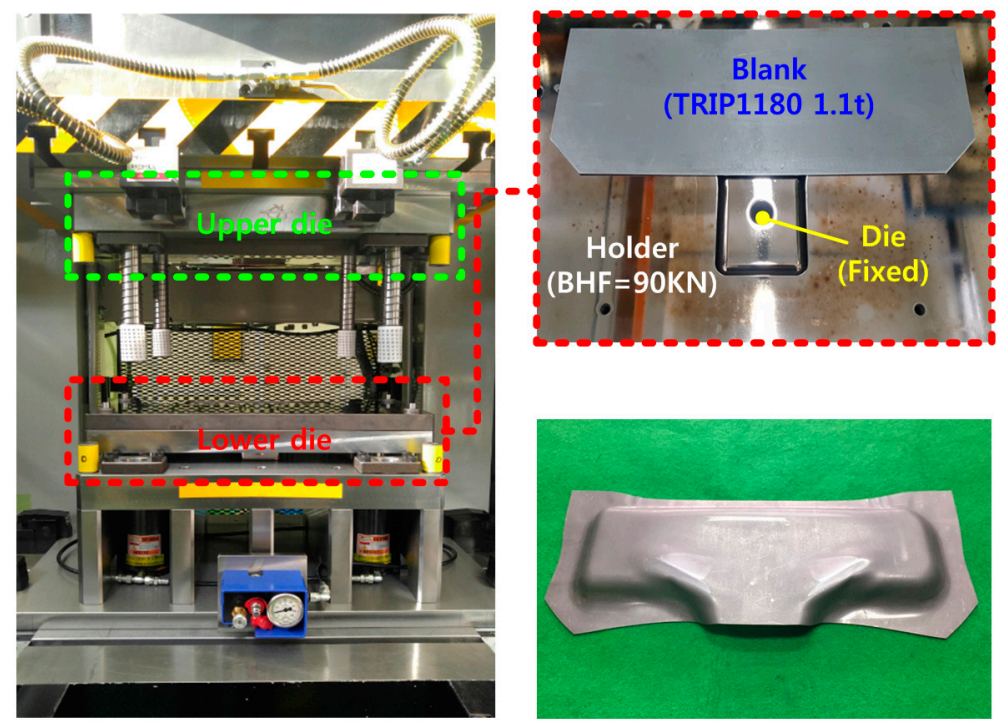

Figure 6. Experimental set-up for T-shape drawing test.

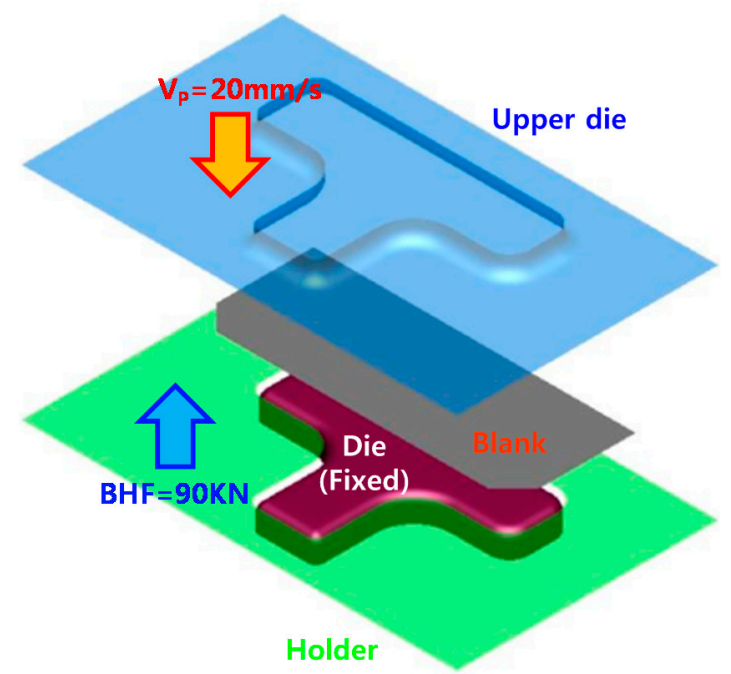

Figure 7. FE model for T-shape drawing test.

\section{Results and discussion}

\subsection{Springback prediction for U-bending test}

In order to investigate springback prediction accuracy according to the constitutive equation, the prediction results of the U-bending test were compared with experimental results. The comparison result is shown in Fig. 8. When the Hill'48 yield function and isotropic hardening model were used, the specimen shapes were different from those observed in the experimental results. However, when the Yld2000-2d yield function and Yoshida-Uemori model were used, the predicted shapes were similar with those of the manufactured parts. 
Springback parameters were employed in this study to quantitatively compare springback. Fig. 9(a) shows the springback parameters for the defined Numisheet' 93 benchmark problem. As previously mentioned, when the Yld2000-2d yield function and Yoshida-Uemori model were used, the prediction accuracy for springback was excellent for various rolling directions, such as $0^{\circ}, 45^{\circ}$, and $90^{\circ}$, as shown in Fig. 9 .

Comparing the results for prediction and experiment of U-bending test, hardening model dominantly influenced on prediction accuracy of springback. When an isotropic hardening model was used, the predicted shape differed greatly from that in the experimental result. However, when the Yoshida-Uemori model was used, the predicted shape was similar to that observed in the experimental results. For the U-bending test, the deformation mode of the sheet was a uniaxial tension mode, and the sheet was deformed with non-linear loading conditions. Anisotropic behavior in the uniaxial tension mode could be described well by the Hill'48 or Yld2000-2d yield functions, whereas hardening behavior with non-linear loading conditions is only described by the YoshidaUemori model, because it effectively considers changes in the elastic modulus due to prestrain, the Bauschinger effect, and transient behavior.

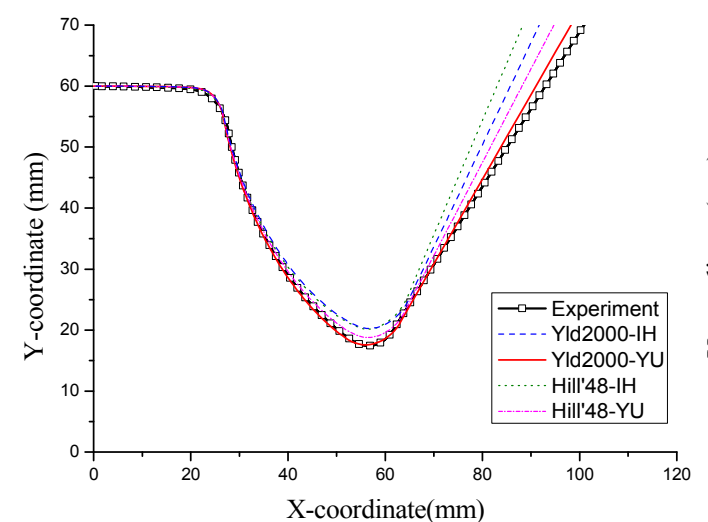

(a) Rolling direction $\left(0^{\circ}\right)$

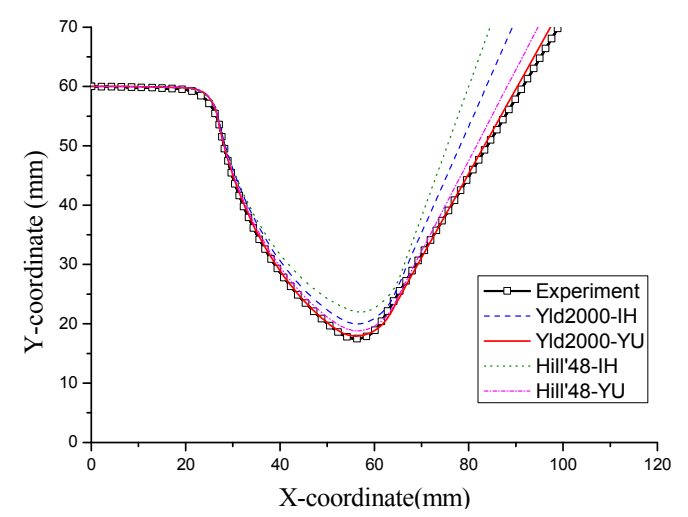

(c) Transverse direction $\left(90^{\circ}\right)$

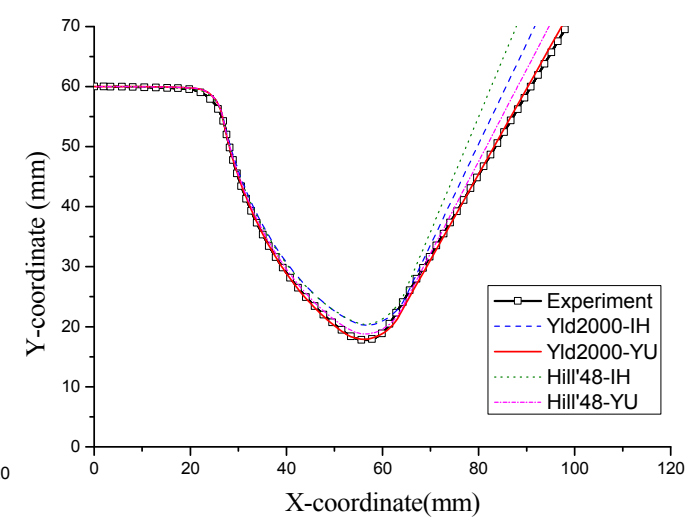

(b) Diagonal direction $\left(45^{\circ}\right)$

Figure 8. Shape comparison between predictions and experiments for U-bending test. 


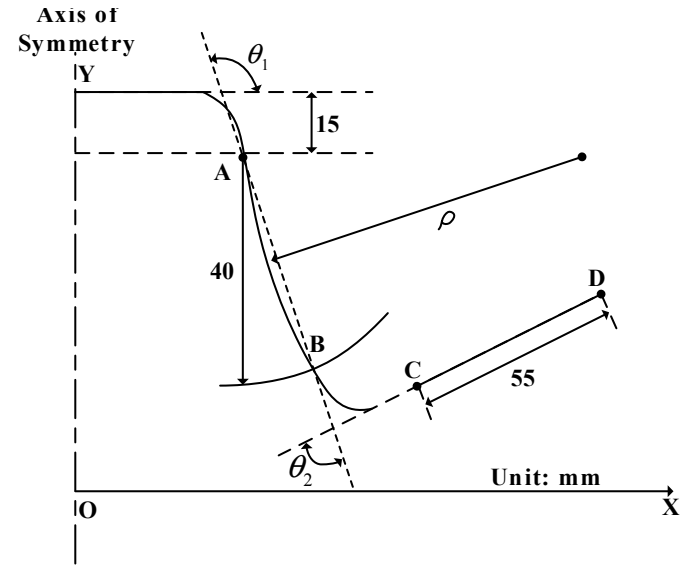

(a) Springback parameters

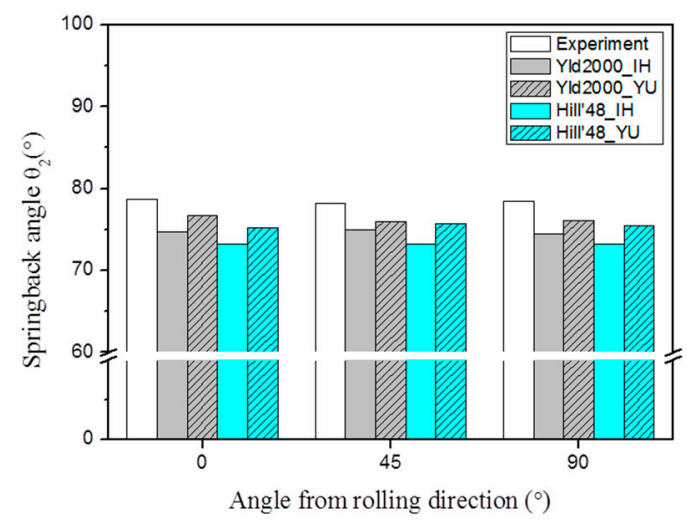

(c) $\theta_{2}$

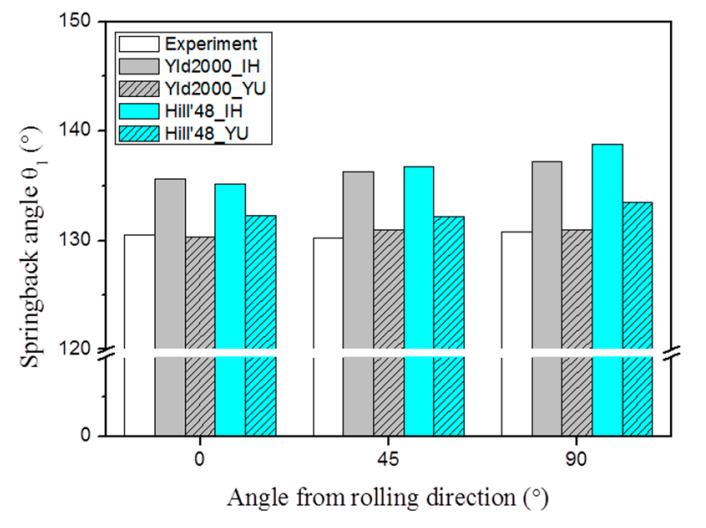

(b) $\theta_{1}$

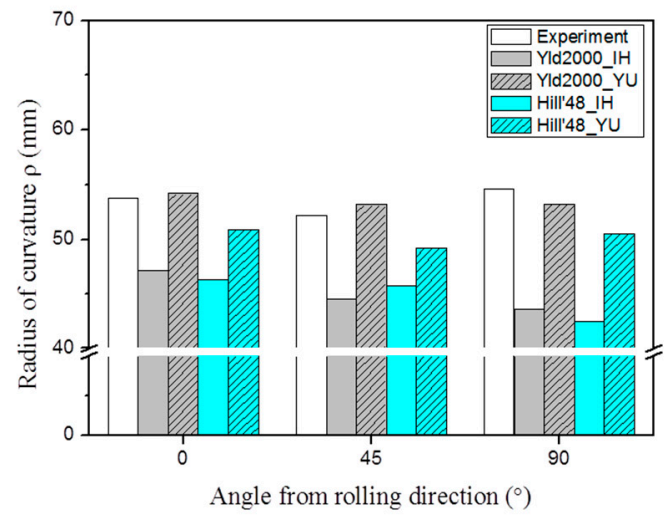

(d) $\rho$

Figure 9. Comparison of springback with springback parameters for U-bending test.

\subsection{Sprinback prediction for T-shape drawing test}

In order to investigate the effects of constitutive equations on springback prediction accuracy, prediction results for the T-shape drawing test were compared with experimental results, as shown in Fig. 10. The springback prediction accuracy for the T-shape drawing had the same tendencies observed for the U-bending test. In other words, the Yld2000-2d yield function and the YoshidaUemori model demonstrated excellent prediction accuracy, with an agreement rate of $82.21 \%$, whereas the Hill' 48 yield function and isotropic hardening model showed low prediction accuracy, with an agreement rate of $73.54 \%$. In this study, the agreement rate was defined as follows:

$$
\text { Agreement rate }=\frac{A_{ \pm 0.5}}{A_{\text {total }}} \times 100(\%)
$$

where $\mathrm{A}_{ \pm 0.5}$ is the satisfied area for an allowable tolerance of $\pm 0.5 \mathrm{~mm}$, and $A_{\text {total }}$ is the total area for the manufactured part.

In comparing the prediction and experimental results for the T-shape drawing test, it can be observed that the yield function dominantly influenced springback prediction accuracy. When the Hill'48 yield function was used, the predicted shape differed greatly from the shape observed in experimental results. However, when Yld2000-2d was used, the predicted shape was similar to that observed in experimental results. In the T-shape drawing test, various deformation modes were represented, and the sheet was deformed with approximately linear loading condition. Hardening behavior in the linear loading condition could be described well by an isotropic or Yoshida-Uemori hardening model, whereas yield behavior for various deformation modes is only described by the Yld2000-2d yield function, because that function considers Rankford values and yield stress according to the rolling direction and biaxial deformation mode. 


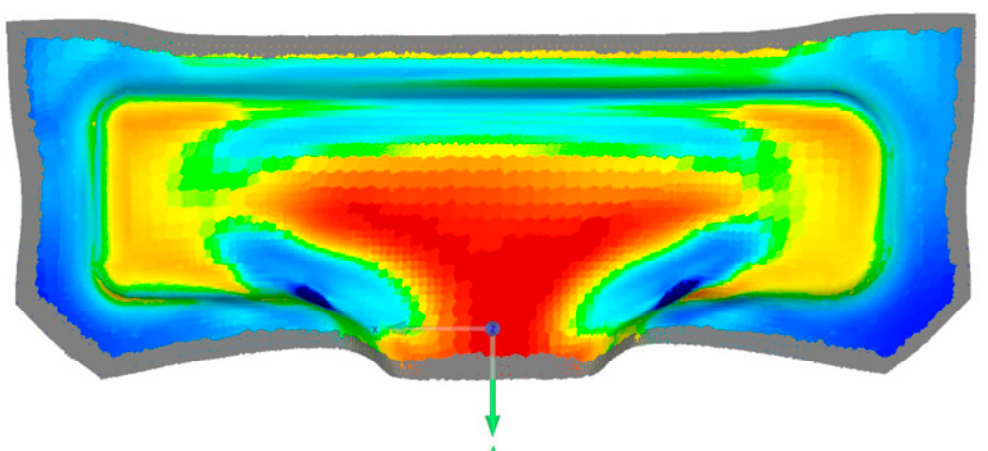

(a) Hill' 48 and isotropic hardening

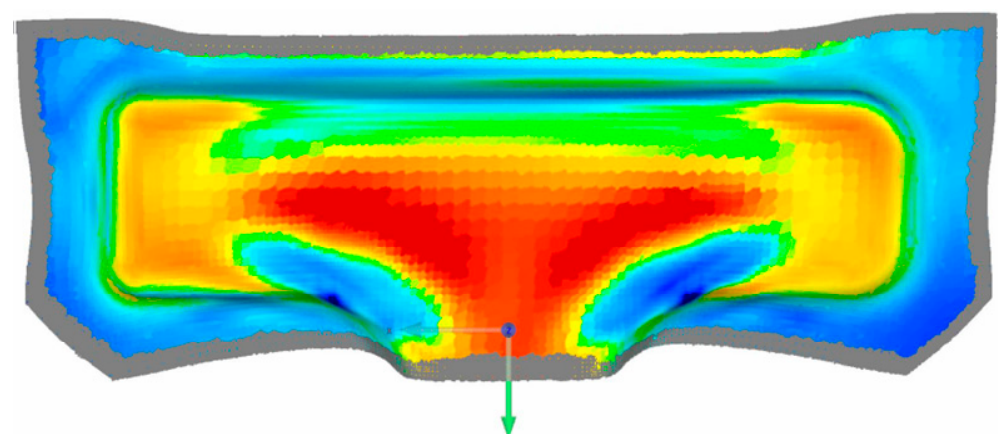

Dim.

(b) Hill'48 and Yoshida-Uemori model

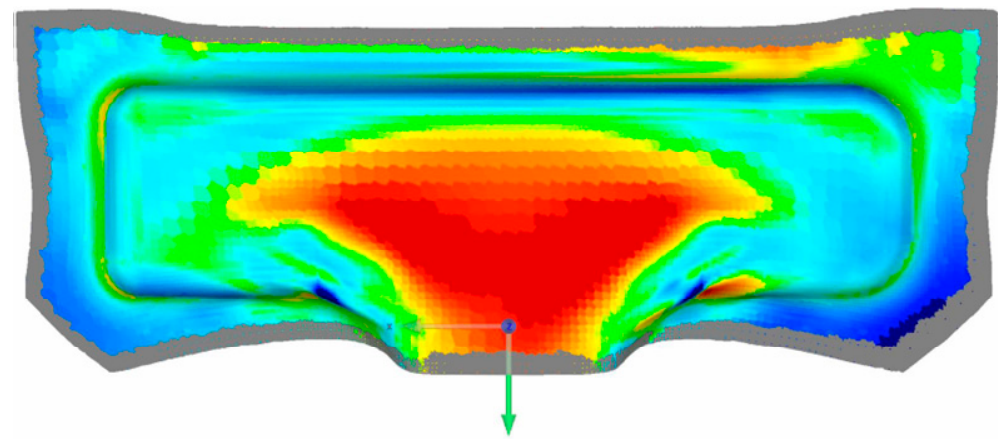

(c) Yld2000-2d and isotropic hardening

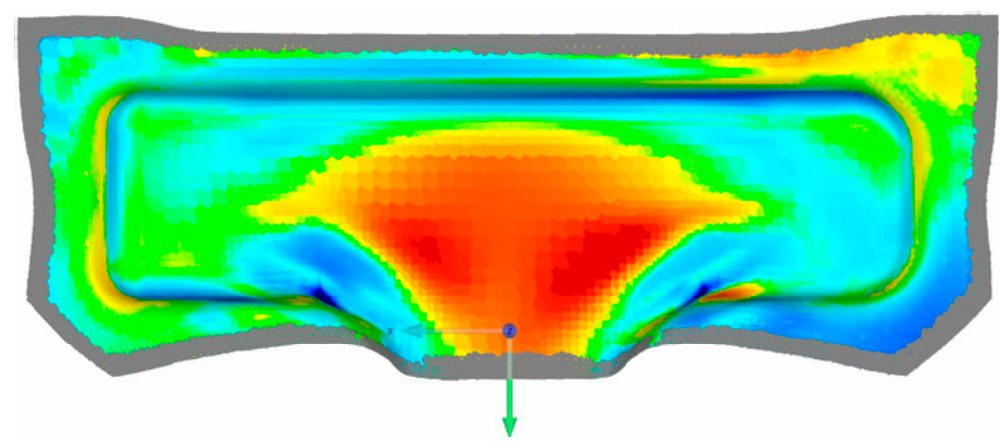

Err.

\begin{tabular}{l}
1.0000 \\
0.9000 \\
0.8000 \\
0.7000 \\
0.6000 \\
0.5000 \\
0.4000 \\
0.3000 \\
0.2000 \\
0.1000 \\
\\
-0.1000 \\
-0.2000 \\
-0.3000 \\
-0.4000 \\
-0.5000 \\
-0.6000 \\
-0.7000 \\
-0.8000 \\
-0.9000 \\
-1.0000 \\
\hline
\end{tabular}

[mm]

(d) Yld2000-2d and Yoshida-Uemori model

Figure 10. Comparison between predictions and experiments for T-shape drawing test. 


\section{Conclusions}

In this study, FEA and experiments were conducted to evaluate the effect of constitutive equa -ations on springback prediction accuracy in the cold stamping of a TRIP1180 sheet. Based on the experimental and analytical results, the following conclusions can be drawn:

1. Uniaxial tension, bulge, tension-compression, and loading-unloading tests were conducted to investigate anisotropy, nonlinear hardening behavior, and changes in the elastic modulus of a TRIP1180 sheet. Based on experimental results, the material constants of various constitutive equations were determined, and were implemented in an FE simulation for forming and springback analysis.

2. FE simulations and experiments were performed to evaluate springback behavior in U-bending and T-shape drawing tests. In both cases, the Yld2000-2d yield function and Yoshida-Uemori model showed excellent prediction accuracy, whereas the Hill'48 yield function and isotropic hardening model showed low prediction accuracy.

3. In the U-bending test, the hardening model dominantly influenced the prediction accuracy of springback, because of its non-linear loading conditions. Hardening behavior with non-linear loading conditions was only described by the Yoshida-Uemori model, because it effectively considered changes in the elastic modulus due to prestrain, the Bauschinger effect, and transient behavior.

4. In the T-shape drawing test, the yield function dominantly influenced the prediction accuracy of springback because of its various deformation modes. Yield behavior with various deformation modes was only described by the Yld2000-2d yield function, because it considered Rankford values and yield stresses according to the rolling direction and biaxial deformation mode.

5. In springback prediction in the cold stamping of AHSS, it is necessary to use an appropriate constitutive equation according to the forming process. In addition, constitutive equations need to accurately describe yield behavior, elastic modulus changes, and hardening behavior in a variety of deformation modes.

Acknowledgments: This work was supported by the Small and Medium Business Administration of Korea (SMBA) grant funded by the Korea government (MOTIE) (No. S2315965).

Author Contributions: Ki-Young Seo and Byung-Min Kim conceived and designed the experiments; Ji Hoon Kim interpreted results; Jae-Hong Kim and Ki-Young Seo wrote and revised the manuscript; Hyunk-Seok Lee measured the experiment specimens and supplied the materials.

Conflicts of Interest: The authors declare no conflict of interest.

\section{References}

1. Hisashi, H.; Nakagawa, T. Recent trends in sheet metals and their formability in manufacturing automotive panels. J. Mater. Process. Technol. 1994, 115, 2-8.

2. Chen, P; Koc, M. Simulation of springback variation in forming of advanced high strength steels. J. Mater. Process. Technol. 2007, 190, 189-198.

3. Chaboche, J.L. Constitutive equations for cyclic plasticity and cyclic viscoplasticity. Int. J. Plast. 1989, 5, 247-302.

4. Lee, M.G.; Chung, K.; Kim, D.; Kim, C.; Wenner, M.L.; Barlat, F. Spring-back evaluation of automotive sheets based on isotropic-kinematic hardening laws and non-quadratic anisotropic yield functions, Part I: theory and formulation. Int. J. Plasticity. 2004.

5. S.L. Zang.; C. Guo.; S. Thuillier.; Lee, M.G. A model of one-surface cyclic plasticity and its application to springback prediction. Int. J. Mech. Sci. 2011, 53, 425-435.

6. R. Larsson.; O. Bjoerklund.; L. Nilsson.; K. Simonsson. A study of high strength steels undergoing non-linear strain paths - experiments and modelling. J. Mater. Process. Technol. 2011, 211, 122-132.

7. P.A. Eggertsen.; K. Mattiasson. On the modeling of the bending-unbending behaviour for accurate springback predictions. Int. J. Mech. Sci. 2009, 51, 547-563. 
8. F. Yoshida. Material models for accurate simulation of sheet metal forming and springback. AIP Conference Proceedings 1252, Pohang, Korea. 2010, 71-78.

9. Y.X. Zhu.; Y.L. Liu.; H. Yang.; H.P. Li. Development and application of the material constitutive model in springback prediction of cold-bending. Mater. Des. 2012, 42, 245-258.

10. R. Hill. A Theory of the Yielding and Plastic Flow of Anisotropic Metals, Proc. R. Soc. Lond. 1948, 193, 281 297.

11. F. Barlat.; J. C. Brem.; J. W. Yoon.; K. Chung.; R. E. Dick.; D. J. Lege.; F. Pourboghrat.; S. H. Choi.; E. Chu. Plane Stress Yield Function for Aluminum Alloy Sheets - Part 1: Theory. Int. J. Plast. 2003, 19, 1297 1319.

12. H. W. Swift. Plastic instability under plane stresses. J. Mech. Phys. Solids. 1952, 1, 1-18.

13. F. Yoshida.; T. Uemori. A Model of Large-strain Cyclic Plasticity Describing the Bauschinger Effect and Workhardening Stagnation. Int. J. Plast. 2002, 18, 661 686.

14. S. Chatti.; R.A. Fathallah. A study of the variations in elastic modulus and its effect on springback prediction. Int. J. Mater. Form. 2014, 7, 19-29.

15. A. Ghaei.; D.E. Green.; A. Aryanpour. Springback simulation of advanced high strength steels considering nonlinear elastic unloading-reloading behavior. Mater. Des. 2015, 88, 461-470.

16. C. Gomes.; O. Onipede.; M. Lovell. Investigation of Springback in High Strength Anisotropic Steels. J. Mater. Process. Technol. 2005, 159, 91 98.

(C) 2017 by the authors. Licensee Preprints, Basel, Switzerland. This article is an open access article distributed under the terms and conditions of the Creative Commons by Attribution (CC-BY) license (http://creativecommons.org/licenses/by/4.0/). 